\title{
Tolerability of a Proprietary Larch Arabinogalactan Extract: A Randomized, Double-Blind, Placebo-Controlled Clinical Trial in Healthy Subjects
}

\author{
Barbara Grube ${ }^{1}$, Heike Stier ${ }^{2}$, Linda Riede ${ }^{2}$, Joerg Gruenwald ${ }^{2}$ \\ ${ }^{1}$ Kurfürstendamm 157/158, 10709 Berlin, Germany; ${ }^{2}$ Analyze \& Realize ag, Waldseeweg 6, 13467 Berlin, Germany. \\ Email: hstier@analyze-realize.com
}

Received September $13^{\text {th }}, 2012$; revised October $13^{\text {th }}, 2012$; accepted October $20^{\text {th }}, 2012$

\begin{abstract}
In a placebo-controlled, double-blind, randomized clinical trial, the tolerability of a proprietary larch arabinogalactan preparation (ResistAid ${ }^{\mathrm{TM}}$ ) was investigated. METHODS: 199 healthy participants were randomly assigned to receive either placebo $(n=98)$ or an arabinogalactan preparation $(n=101)$ over a period of 12 weeks. As safety parameters the total number of adverse events, changes in various biochemical and laboratory parameters as well as the global evaluation of tolerability by investigator and subjects compared to placebo were evaluated. RESULTS: In total 16 adverse events were observed in 16 subjects, with no difference between the arabinogalactan and the placebo group $(\mathrm{p}=0.935)$. There were no differences in the mean changes of the measured biochemical and laboratory parameters. The tolerability of the arabinogalactan extract was rated as "very good" or "good" by the investigators for $99 \%$ of the subjects and by $98 \%$ subjects in self-assessment with no statistical differences to placebo. CONCLUSION: The measured parameters as well as the evaluation of the tolerability by the investigators and the subjects demonstrate a very good tolerance profile of the proprietary arabinogalactan extract with no differences to placebo when taken for 12 weeks.
\end{abstract}

Keywords: Randomized Placebo-Controlled Study; Proprietary Larch Arabinogalactan; Safety; Tolerability; ResistAid $^{\mathrm{TM}}$

\section{Introduction}

Arabinogalactans are long, densely branched, polysaccharides with molecular weight ranging from 10,000 to 120,000 Daltons consisting of the two monomers arabinose and galactose. They are mostly present in glycoprotein form, bound to a protein spine of threonine, proline or serine (arabinogalactan protein). In nature, arabinogalactans are found in microbial systems and plants. As part of the cell walls they can be found in several plants with immune-enhancing properties like Echinacea purpurea or Curucuma long but also many other plants such as carrots, radish, pears, or maize [1-4]. They are a very heterogeneous class of molecules, depending on the herbal source.

The major commercial source, however, is the larch tree. All arabinogalactans extracted from Larix spp. are water soluble, nitrogen-free highly branched molecules composed of galactose and arabinose units in a 6:1 ratio, with trace amounts of glucuronic acid. Larch arabinogalactan has a galactan backbone that features $\beta(1,3)$ linkages and galactose $\beta(1,6)$ and arabinose $\beta(1,6$ and 1,3$)$ sugar side chains [2]. In contrast to larch typically the concentrations of arabinogalactan in non-larch arabinogalactan other sources is low $(0.5 \%-2 \%)$ whereas in larch arabinogalactan used for ResistAid ${ }^{\mathrm{TM}}$ the proportion is approximately $85 \%$. Further the larch arabinogalactan is not linked to protein.

Arabinogalactan is a dietary fiber, which resists digestion by alimentary tract enzymes and reaches the colon, which makes it comparable to inulin and $\beta$-glucan. Various preparations of arabinogalactan are marketed as ingredients for functional food or as dietary supplements. In vitro and in vivo studies, as well as clinical trials have shown an immune enhancing activity of arabinogalactan preparations [5-7].

The present study examined the tolearability of a proprietary arabinogalactan extract from larch tree (Resist$\mathrm{Aid}^{\mathrm{TM}}$ ) as compared to placebo in healthy adults over a period of 12 weeks. ResistAid ${ }^{\mathrm{TM}}$ is a combination of arabinogalactan and natural bioactive polyphenols extracted from larch trees. It is approved by the US FDA as a direct food additive. It is a brown powder with a neutral taste and a fine pine-like aroma that dissolves quickly in water or juice. The larch arabinogalactan in this product 
has been approved as "Generally Recognized as Safe" (GRAS) by the FDA (GRAS Notice No. GRN000084).

\section{Materials and Methods}

The study was approved by the local ethics advisory committee (Charité Berlin, Germany). The study was conducted according to the Declaration of Helsinki, the ICH-GCP guidelines and EU recommendations (CPMP/ ICH/135/95). It is registered at International Standard Randomised Controlled Trial Number Register, www.isrctn.org (ISRCTN41183655).

\subsection{Study Design}

This study was conducted as a prospective multi-centric, randomized, double-blind, placebo-controlled study in healthy outpatient subjects between October 2010 and May 2011. The subjects were enrolled at 6 study sites in Germany.

\subsection{Study Population}

204 healthy subjects were included in this nutritional study. They had to meet the following inclusion criteria: age between 18 and 70 years (child-bearing females had to agree to use appropriate birth control methods), self-reported incidence of at least 3 upper airway infections in a 6 months period, written informed consent of the subject. The exclusion criteria were as follows: acute or chronic upper airways disease, suspected influenza or swine flu, vaccination against influenza or swine flu within 21 days before the study start, BMI $>30$, clinically significant abnormal laboratory parameters, known sensibility to one of the ingredients of the study product, immune deficiency diseases, severe organ or systemic disorders, body temperature $\geq 38^{\circ} \mathrm{C}$, pregnancy or nursing, use of immunosuppressing or immunostimulating agents, incidence of alcohol, medication or drug abuse, use of pre- and probiotics, participation in another clinical study at or within 30 days before study start, as well as inability to comply with study requirements. Participants were instructed not to change their eating habits.

\subsection{Randomization and Blinding Procedure}

This study was conducted double-blind; both the participants and investigators were unaware of the treatment the participants were receiving. An independent external statistician created the randomization code (block randomization; block size 4). Group assignment to the study arms was performed during the first examination.

\subsection{Intervention}

In this study, the tolerability of the food supplement Re-
sistAid $^{\mathrm{TM}}$ (provided by Lonza Ltd., Basel, Switzerland) was examined. It is a proprietary water-based extract from larch tree (Latrix laricinia). The placebo product was Maltrin ${ }^{\circledR}$ M100 (Maltodextrin, Grain Processing Corporation, Muscatine, USA). Verum and placebo were identical in appearance and taste.

Subjects were randomly assigned to receive daily a to-

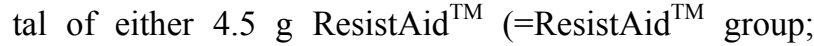
RAG) or placebo (placebo group; PG), respectively; over a course of 12 weeks. Subjects were instructed to dissolve the content of a sachet with the investigational product (verum or placebo) in approximately $100-150$ $\mathrm{mL}$ of liquid and take the prepared drink at breakfast. During the study period of 12 weeks, a total of 3 visits were performed: at baseline (visit 1), after 6 weeks (visit 2 ) and at the end after 12 weeks (visit 3) in the practices of the six investigators. Eating habits were recorded in a diet diary at start and end of the study.

Compliance was checked by counting returned unused sachets. It was considered sufficient if $>75 \%$ and $<125 \%$ of the sachets were consumed.

\subsection{Safety Measurements}

The safety of the investigational study product compared to placebo was assessed by documentation of adverse events throughout the study. At each visit, the investigator questioned the subject if any adverse events (AEs) had occurred, rated the seriousness and intensity of the AE. Further the investigators judged as to whether the observed $\mathrm{AE}$ had a causal relation to the intake of the investigational product.

Routine clinical parameters (heart rate, blood pressure, body temperature) were assessed at each examination. The laboratory parameters hemoglobin, hematocrit, erythrocytes, leukocytes, thrombocytes, alanine aminotransferase, aspartate aminotransferase, gamma-glutamyltransferase, total bilirubin, creatinine, and uric acid were assessed at baseline and at the end of the intervenetion.

At the end of the study the tolerability of the product was evaluated by the investigator and the subject as "very good", "good", "moderate", or "poor".

\subsection{Statistical Analysis}

All the variables contained in the data collection were presented descriptively using their statistical key data or their frequency distribution and statistically analyzed in view of the group specific differences $\left(\mathrm{p}_{\chi}{ }^{2}\right.$-value). The Mann-Whitney U-test was employed to test for between-groups comparison $\left(\mathrm{p}_{\mathrm{u}}\right)$. The statistical analyses were carried out on a full analysis set (FAS). 


\section{Results}

\subsection{Study Population}

Between October 2010 and February 2011, 204 subjects were enrolled. Three subjects had laboratory values above the limit defined in exclusion criteria and were thus excluded from the intent to treat (ITT) population. Additionally two subjects had no data except baseline and were also excluded from FAS population.

Of the 199 subjects in the FAS population, $65(32.7 \%)$ were men and 134 (67.3\%) were women. 198 (99.5\%) were classified as "Caucasian" and 1 subject $(0.5 \%)$ as "Asian". The measured baseline characteristics of the subjects are provided in Table $\mathbf{1}$ and demonstrate the comparability of the two study groups.

\subsection{Assessment of Adverse Events}

During the study, 16 adverse events (AEs) were documented in 16 subjects (16 of 204; $7.8 \%$ ), 8 in each study group. There was no difference between the groups in the proportion of subjects with AEs $\left(\mathrm{p}_{\mathrm{Chi}}=0.935\right)$. The individual AEs are listed in Table 2.

According to the investigators, only one AE (soft stool 30 minutes after intake of investigational product in fasting state) was "probably related" to the intake of the investigational product (placebo). For all other AEs the relation to the intake of ResistAid ${ }^{\mathrm{TM}}$ or placebo was stated to be "none", "unlikely", or "not assessable". There was no significant difference between the groups with regard to the causality to the intake of investigational product $\left(\mathrm{p}_{\text {Fisher }}=1.000\right)$.

One of the reported AEs was judged as a serious adverse event (pneumonia right lower lobe) and led to study termination. This SAE, however, was not related to the intake of the investigational product (ResistAid $^{\mathrm{TM}}$ group).

All but one (pneumonia right lower lobe) of the AEs

Table 1. Baseline characteristics of the study population.

\begin{tabular}{cccc}
\hline $\begin{array}{c}\text { Parameter at } \\
\text { Baseline }\end{array}$ & $\begin{array}{c}\text { RAG }(\mathrm{N}=101) \\
\text { MV } \pm \mathrm{SD}\end{array}$ & $\begin{array}{c}\text { PG }(\mathrm{N}=98) \\
\mathrm{MV} \pm \mathrm{SD}\end{array}$ & $\begin{array}{c}\mathrm{p}_{\mathbf{u}} \\
\text { value }\end{array}$ \\
\hline Age [years] & $42.0 \pm 14.9$ & $42.4 \pm 15.8$ & 0.911 \\
Height [cm] & $170.7 \pm 9.0$ & $171.6 \pm 7.7$ & 0.417 \\
Weight $[\mathrm{kg}]$ & $69.5 \pm 12.5$ & $70.9 \pm 11.2$ & 0.240 \\
BMI $\left[\mathrm{kg} / \mathrm{m}^{2}\right]$ & $23.7 \pm 3.0$ & $24.0 \pm 2.7$ & 0.487 \\
Syst. bp $[\mathrm{mmHg}]$ & $123.9 \pm 15.8$ & $123.2 \pm 14.6$ & 0.742 \\
Diast. bp [mmHg] & $78.8 \pm 7.3$ & $78.2 \pm 6.8$ & 0.636 \\
Heart rate $[1 / \mathrm{min}]$ & $71.4 \pm 7.0$ & $71.2 \pm 6.9$ & 0.795 \\
Body temperature $\left[{ }^{\circ} \mathrm{C}\right]$ & $36.6 \pm 0.3$ & $36.5 \pm 0.3$ & 0.188 \\
\hline
\end{tabular}

RAG: ResistAid ${ }^{\mathrm{TM}}$; PG: placebo group; MV: mean value; SD: standard deviation; BMI: body mass index; Syst. bp: systolic blood pressure; diast. bp: diastolic blood pressure. were judged to be of "mild" or "moderate" intensity.

\subsection{Assessment of Clinical Parameters}

Routine clinical parameters (heart rate, blood pressure, body temperature) were determined at each examination. For the three parameters heart rate, body temperature and diastolic blood pressure, no differences between the two treatments were observed. However, the measured systolic blood pressure showed differences between the ResistAid $^{\mathrm{TM}}$ group and the placebo group (see Table 3).

Table 2. Overview about the documented adverse events.

\begin{tabular}{l} 
AEs in the ResistAid ${ }^{\mathrm{TM}}$ group \\
\hline Gastrointestinal infection (cramps, diarrhea, vomiting) \\
Cramp-like chest pain, local and radiating \\
Purulent bronchitis (abnormal laboratory values at final visit) \\
Cervical syndrome \\
Urinary infection \\
Pneumonia right lower lobe \\
Abnormal laboratory values at final visit (high alanine und aspartate \\
aminotransferase) \\
Gastrointestinal infection (cramps, diarrhea) \\
\hline HEs in the placebo group \\
Hypoglycemia with cold sweat \\
Urinary infection \\
Abnormal laboratory values at final visit (high gamma-glutamyl \\
transferase) \\
Soft stool 30 minutes after intake of investigational product in fast- \\
ing state \\
Hay fever \\
Gastroenteritis
\end{tabular}

Table 3. Changes in blood pressure.

\begin{tabular}{|c|c|c|c|}
\hline & $\operatorname{RAG}(\mathrm{N}=101)$ & $\mathrm{PG}(\mathrm{N}=98)$ & \multirow{2}{*}{$\mathrm{p}_{\mathrm{u}}$ value } \\
\hline & $\mathrm{MV} \pm \mathrm{SD}$ & $\mathrm{MV} \pm \mathrm{SD}$ & \\
\hline \multicolumn{4}{|c|}{ Systolic blood pressure [Mmhg] } \\
\hline Changes V1 - V2 & $3.5 \pm 11,8$ & $1.1 \pm 9.7$ & 0.070 \\
\hline (pt-value) & $(0,004)$ & $(0.267)$ & \\
\hline Changes V1 - V3 & $2.9 \pm 11.0$ & $1.5 \pm 11.1$ & 0.105 \\
\hline (pt-value) & $(0.009)$ & $(0.195)$ & \\
\hline \multicolumn{4}{|c|}{ Diastolic blood pressure [mmHg] } \\
\hline Changes V1 - V2 & $1.3 \pm 7.4$ & $0.2 \pm 6.5$ & 0.370 \\
\hline (pt-value) & $(0.087)$ & $(0,755)$ & \\
\hline Changes V1 - V3 & $0.7 \pm 6.6$ & $0.3 \pm 6.7$ & 0.790 \\
\hline (pt-value) & $(0.270)$ & $(0.632)$ & \\
\hline
\end{tabular}

RAG: ResistAid ${ }^{\mathrm{TM}}$ group; PG: placebo group; MV: mean value; SD: standard deviation; V1: visit 1; V2; visit 2; V3: visit 3. 
The systolic blood pressure was significantly lower after 6 and 12 weeks of treatment, respectively, compared to baseline, in the active but not in the placebo group.

\subsection{Assessment of Laboratory Parameters}

All the measured laboratory parameters were analyzed for changes from Visit 1 to Visit 3. Neither at baseline nor at the end of the study did the two study populations differ in any of the parameters.

Further, there were no statistical differences in the mean changes of the parameters from Visit 1 to Visit 3. All the observed changes in individual subjects were stated by the investigators to be of no clinical relevance (Table 4).

Table 4. Changes in laboratory parameters during the study.

\begin{tabular}{|c|c|c|c|c|c|}
\hline & & V1 & V3 & Difference V3 - V1 & $\mathrm{p}$ value V1 - V3 \\
\hline & $\mathrm{N}$ & $\mathrm{MV} \pm \mathrm{SD}$ & $\mathrm{MV} \pm \mathrm{SD}$ & $\mathrm{MV} \pm \mathrm{SD}$ & \\
\hline \multicolumn{6}{|c|}{ Haemoglobin [g/dL] } \\
\hline RAG & 100 & $13.92 \pm 1.28$ & $13.83 \pm 1.21$ & $-0.09 \pm 1.17$ & \multirow{2}{*}{0.646} \\
\hline PG & 97 & $13.80 \pm 1.18$ & $13.82 \pm 1.15$ & $0.02 \pm 1.02$ & \\
\hline \multicolumn{6}{|c|}{ Haematocrit [\%] } \\
\hline RAG & 100 & $42.4 \pm 3.6$ & $42.3 \pm 3.4$ & $-0.1 \pm 3.6$ & \multirow{2}{*}{0.658} \\
\hline PG & 97 & $42.0 \pm 3.2$ & $42.2 \pm 3.2$ & $0.2 \pm 2.7$ & \\
\hline \multicolumn{6}{|c|}{ Erythrocytes [Tpt/L] } \\
\hline RAG & 100 & $4.66 \pm 0.41$ & $4.66 \pm 0.38$ & $-0.01 \pm 0.38$ & \multirow{2}{*}{0.824} \\
\hline PG & 97 & $4.61 \pm 0.37$ & $4.64 \pm 0.36$ & $0.03 \pm 0.29$ & \\
\hline \multicolumn{6}{|c|}{ Thrombocytes $[\mathrm{Gpt} / \mathrm{L}]$} \\
\hline RAG & 100 & $261.6 \pm 55.5$ & $248.5 \pm 59.2$ & $-13.1 \pm 57.0$ & \multirow{2}{*}{0.270} \\
\hline PG & 97 & $264.3 \pm 58.0$ & $260.9 \pm 56.3$ & $-3.4 \pm 45.8$ & \\
\hline \multicolumn{6}{|c|}{ Leukocytes [Gpt/L] } \\
\hline RAG & 100 & $7.20 \pm 2.16$ & $6.88 \pm 2.10$ & $-0.32 \pm 1.84$ & \multirow{2}{*}{0.648} \\
\hline PG & 97 & $6.96 \pm 1.96$ & $6.78 \pm 1.88$ & $-0.18 \pm 1.88$ & \\
\hline \multicolumn{6}{|c|}{ ALAT $[\mathrm{U} / \mathrm{L}]$} \\
\hline RAG & 101 & $24.0 \pm 10.5$ & $26.2 \pm 15.7$ & $2.2 \pm 15.1$ & \multirow{2}{*}{0.678} \\
\hline PG & 97 & $23.6 \pm 9.3$ & $24.2 \pm 10.7$ & $0.7 \pm 10.1$ & \\
\hline \multicolumn{6}{|c|}{$\operatorname{ASAT}[\mathrm{U} / \mathrm{L}]$} \\
\hline RAG & 101 & $24.6 \pm 7.6$ & $27.8 \pm 11.7$ & $3.2 \pm 10.2$ & \multirow{2}{*}{0.788} \\
\hline PG & 97 & $25.4 \pm 7.0$ & $28.1 \pm 16.6$ & $2.7 \pm 15.3$ & \\
\hline \multicolumn{6}{|c|}{ GGT [U/L] } \\
\hline RAG & 101 & $27.5 \pm 19.9$ & $25.8 \pm 16.6$ & $-1.7 \pm 14.8$ & \multirow{2}{*}{0.617} \\
\hline PG & 97 & $23.9 \pm 12.7$ & $26.7 \pm 24.7$ & $2.8 \pm 23.9$ & \\
\hline \multicolumn{6}{|c|}{ Bilirubin $[\mathrm{mg} / \mathrm{dL}]$} \\
\hline RAG & 101 & $0.59 \pm 0.25$ & $0.62 \pm 0.31$ & $0.03 \pm 0.29$ & \multirow{2}{*}{0.535} \\
\hline PG & 97 & $0.61 \pm 0.35$ & $0.59 \pm 0.32$ & $-0.01 \pm 0.28$ & \\
\hline \multicolumn{6}{|c|}{ Creatinine $[\mathrm{mg} / \mathrm{dL}]$} \\
\hline RAG & 101 & $0.79 \pm 0.14$ & $0.79 \pm 0.14$ & $-0.01 \pm 0.11$ & \multirow{2}{*}{0.709} \\
\hline PG & 97 & $0.77 \pm 0.14$ & $0.78 \pm 0.14$ & $0.01 \pm 0.12$ & \\
\hline \multicolumn{6}{|c|}{ Uric acid $[\mathrm{mg} / \mathrm{dL}]$} \\
\hline RAG & 101 & $4.76 \pm 1.23$ & $4.71 \pm 1.29$ & $-0.05 \pm 0.94$ & \multirow{2}{*}{0.873} \\
\hline PG & 97 & $4.89 \pm 1.44$ & $4.87 \pm 1.31$ & $-0.02 \pm 1.02$ & \\
\hline
\end{tabular}

RAG: ResistAid ${ }^{\mathrm{TM}}$ group; PG: placebo group; MV: mean value; SD: standard deviation; V1: visit 1; V3: visit 3; ALAT: alanine aminotransferase; ASAT: aspartate aminotransferase; GGT: gamma-glutamyl transferase. 


\subsection{Global Assessment of Tolerability}

There was no statistical difference in rating of the tolerability of ResistAid ${ }^{\mathrm{TM}}$ compared to that of placebo either by the investigator $\left(\mathrm{p}_{\mathrm{Chi}}=0.417\right)$ or by the subjects $\left(\mathrm{p}_{\mathrm{Chi}}\right.$ $=0.487$ ); Table 5. The tolerability of ResistAidTM was rated as "very good" or "good" by the investigators for $99 \%$ of the subjects and by $98 \%$ subjects in self-assessment. For placebo, the tolerability was rated as "very good" or "good" by the investigators for $100 \%$ of the subjects and by $98.9 \%$ subjects in self assessment. In most cases, the rating of the investigators and the subjects were identical.

\section{Discussion}

In this nutritional study the tolerability of the daily intake of $4.5 \mathrm{~g} \mathrm{ResistAid}^{\mathrm{TM}}$ compared to placebo was addressed in 199 healthy subjects. The results of this study demonstrate that the intake of the proprietary arabinogalactan ResistAid $^{\mathrm{TM}}$ over 12 weeks can be considered safe. There were no differences between the ResistAid ${ }^{\mathrm{TM}}$ and the placebo group in the mean changes of the measured laboratory parameters. Furthermore, there were no differences concerning the incidence of adverse events. Causality assessment of the adverse events by the investigator showed that none of the AEs was related to the intake of the arabinogalactan preparation. Also when the rated tolerability between ResistAid ${ }^{\mathrm{TM}}$ and placebo was compared no statistical differences have been found.

ResistAid ${ }^{\mathrm{TM}}$ was rated as "very good" or "good" by $98 \%$ of the subjects and for $99 \%$ of the subjects by the investigator. It is worth mentioning that the systolic blood pressure decreases significantly from baseline to the end of the study within the arabinogalactan preparation treated group but not in the placebo group. This effect was already observed at the second examination (after 6 weeks).

Arabinogalactan isolated form larch is approved by the FDA for food use at levels commonly used for food ad- ditives (Generally Recognized as Safe-GRAS). It can be used as an emulsifier, stabilizer, binder or bodying agent in essential oils. Acute toxicity studies in rats demonstrated a very good safety profile, better than methyl cellulose. Like arbabinogalactan, methyl cellulose is an important emulsifier used by the food industry e.g. in ice cream.

Acute toxicity studies in rat or mice showed that even $5000 \mathrm{mg} / \mathrm{kg}$ body weight led to no symptoms of toxicity. In long term studies $500 \mathrm{mg} / \mathrm{kg}$ body weight over 90 days showed no toxic effects (for review see [2,3]).

Several human trials have also been performed with larch arabinogalactan [8-10]. Even in concentration as high as $30 \mathrm{~g}$ /day over a period of three weeks intake of arabinogalactan was very well tolerated [8]. The main adverse effects stated for arabinogalactan are gastrointestinal complaints such as bloating and flatulence [1]. This might be related to the fact that arabinogalactan is not digested by the intestinal enzymes but serves as a prebiotic for the gastrointestinal bacteria [11]. This in turn may alter them with time of consumption.

When arabinogalactan was administered to dogs (various preparations and concentration from $0.55 \mathrm{~g} / \mathrm{d}$ to $1.65 \mathrm{~g} / \mathrm{d}$ ), an increase of white blood cells has been observed [11]. However, none of the human trials, where comparable doses were applied, showed any significant changes in blood cells. For example, when arabinogalactan was applied for 4 weeks in 3 different concentrations ranging from $1.5-4.5 \mathrm{~g} /$ day no changes in monocyte count was detected [10]. In another randomized, double blind, placebo controlled pilot study [12], a low dose of larch arabinogalactan (1.5 g/day) was tested; again, a 4-week supplementation period did not alter cell counts of total blood leukocytes, neutrophils, lymphocytes, or monocytes. In a more recently published trial, using the same arabinogalactan preparation as in this presented study, the daily intake of $4.5 \mathrm{~g}$ arabinogalactan over 72 days did not lead to any changes in white blood cells.

The results of all these studies performed with larch

Table 5. Global assessment of tolerability, investigators.

\begin{tabular}{|c|c|c|c|c|c|c|c|c|}
\hline \multirow{3}{*}{$\begin{array}{c}\text { Global assessment } \\
\text { of tolerability }\end{array}$} & \multicolumn{4}{|c|}{ By investigator } & \multicolumn{4}{|c|}{ By subjects } \\
\hline & \multicolumn{2}{|c|}{ RAG (N = 99) } & \multicolumn{2}{|c|}{$\mathrm{PG}(\mathrm{N}=97)$} & \multicolumn{2}{|c|}{ RAG (N = 99) } & \multicolumn{2}{|c|}{$\mathrm{PG}(\mathrm{N}=95)$} \\
\hline & $\mathrm{N}$ & $\%$ & $\mathrm{~N}$ & $\%$ & $\mathrm{~N}$ & $\%$ & $\mathrm{~N}$ & $\%$ \\
\hline Very good & 64 & 64.6 & 68 & 70.1 & 59 & 58.4 & 65 & 68.4 \\
\hline Good & 34 & 34.3 & 29 & 29.9 & 38 & 37.6 & 29 & 30.5 \\
\hline Moderate & 0 & 0.0 & 0 & 0.0 & 2 & 2.0 & 1 & 1.1 \\
\hline Poor & 1 & 1.0 & 0 & 0.0 & 0 & 0.0 & 0 & 0.0 \\
\hline $\mathrm{p}_{\text {Chi }}$ value & \multicolumn{4}{|c|}{0.417} & \multicolumn{4}{|c|}{0.487} \\
\hline
\end{tabular}

RAG: ResistAid ${ }^{\mathrm{TM}}$ group; PG: placebo group; N: number. 
arabinogalactan, indicate that arabinogalactan is safe for human consumption. In summary, the intake of ResistAid ${ }^{\mathrm{TM}}$ over a time period of 12 weeks can be considered as safe as placebo.

\section{Acknowledgements}

The study was funded by Lonza Ltd. (Basel, Switzerland). Barbara Grube was the principal investigator of the study, the other authors are employees of the CRO coordinating the clinical trials. The authors declare no conflict of interest.

\section{REFERENCES}

[1] Natural Standard, "Arabinogalactan," Natural Standard Monograph, 2010, pp. 1-16.

[2] P. D'Adamo, "Larch Arabinogalactan," Journal of Naturopathic Medicine, Vol. 6, No. 1, 1996, pp. 33-37.

[3] G. S. Kelly, "Larch Arabinogalactan: Clinical Relevance of a Novel Immune-Enhancing Polysaccharide," Alternative Medicine Review, Vol. 4, No. 2, 1999, pp. 96-103.

[4] B. Classen, S. Thude, W. Blaschek, M. Wack and C. Bodinet, "Immunomodulatory Effects of Arabinogalactan-Proteins from Baptisia and Echinacea," Phytomedicine, Vol. 13, No. 9-10, 2006, pp. 688-694. doi:10.1016/j.phymed.2005.10.004

[5] J. Hauer and F. A. Anderer, "Mechanism of Stimulation of Human Natural Killer Cytotoxicity by Arabinogalactan from Larix Occidentalis," Cancer Immunol Immunother, Vol. 36, No. 4, 1993, pp. 237-244. doi:10.1007/BF01740905
[6] E. M. Choi, A. J. Kim, Y. O. Kim and J. K. Hwang, "Immunomodulating Activity of Arabinogalactan and Fucoidan in Vitro," Journal of Medicinal Food, Vol. 8, No. 4, 2005, pp. 446-453. doi:10.1089/jmf.2005.8.446

[7] M. Nantz, A. Painter, E. Parker, C. McGill and S. Percival, "Evaluation of Arabinogalactan's Effect on Human Immunity," The FASEB Journal, Vol. 15, No. 4, 2001, p. 633.

[8] R. R. Robinson, J. Feirtag and J. L. Slavin, "Effects of Dietary Arabinogalactan on Gastrointestinal and Blood Parameters in Healthy Human Subjects," Journal of the American College of Nutrition, Vol. 20, No. 4, 2001, pp. 279-285.

[9] J. K. Udani, B. B. Singh, M. L. Barrett and V. J. Singh, "Proprietary Arabinogalactan Extract Increases Antibody Response to the Pneumonia Vaccine: A Randomized, Double-Blind, Placebo-Controlled, Pilot Study in Healthy Volunteers," Nutrition Journal, Vol. 9, 2010, p. 32.

[10] L. S. Kim, P. M. Burkholder and R. F. Waters, "Effects of Low-Dose Larch Arabinogalactan from Larix Occidentalis: A Randomized, Double-Blind, Placebo-Controlled Pilot Study," Journal of Evidence-Based Complementary \& Alternative Medicine, Vol. 7, No. 3, 2002, pp. 221-229.

[11] C. M. Grieshop, E. A. Flickinger and G. C. Fahey Jr., "Oral Administration of Arabinogalactan Affects Immune Status and Fecal Microbial Populations in Dogs," Journal of Nutrition, Vol. 132, No. 3, 2002, pp. 478-482.

[12] L. S. Kim, R. F. Waters and P. M. Burkholder, "Immunological Activity of Larch Arabinogalactan and Echinacea: A Preliminary, Randomized, Double-Blind, Placebo-Controlled Trial," Alternative Medicine Review: A Journal of Clinical Therapeutic, Vol. 7, No. 2, 2002, pp. 138-149. 